\title{
Leveraging Medication Safety through Mobile Computing: Decision Support and Guidance Services for Adverse Drug Event Prevention
}

\author{
Vassilis Koutkias, Vassilis Kilintzis, Nikolaos Beredimas, and Nicos Maglaveras \\ Laboratory of Medical Informatics \\ Medical School, Aristotle University of Thessaloniki \\ Thessaloniki, Greece \\ \{bikout, billyk\}@med.auth.gr, beredim@auth.gr, nicmag@med.auth.gr
}

\begin{abstract}
In this paper, we present an approach for ubiquitous and pervasive access to guidance and decision support services for medication safety targeting patients/citizens and healthcare professionals, respectively. We particularly focus on Adverse Drug Event (ADE) prevention by exploiting a novel decision support framework through mobile technologies. The main aspect of our work involved tailoring the designed services to the mobile terminal and the respective user interaction. For example, we considered the technical features supported by modern mobile phones, such as Augmented Reality (AR), barcode scanning, and Near-Field Communication (NFC) tagging for identifying the drugs of interest, in order to avoid difficulties and errors in keying-in drug names by the user. We illustrate the applicability and virtue of the proposed approach via a mobile application that has been particularly designed to meet these goals.
\end{abstract}

Keywords-Patient Safety; Medication; Adverse Drug Event Prevention; Decision Support and Guidance; Mobile Computing; Smartphones.

\section{INTRODUCTION}

Patient safety is a major challenge worldwide [1]. In particular, medication safety risks such as Adverse Drug Events (ADEs) constitute an important public health issue causing, besides patient harm, considerable extra healthcare costs [2]. Information and Communication Technologies (ICT) may help reducing medication risks by providing healthcare professionals and patients with access to relevant knowledge and services (guidelines, recommendations, alerts, etc.) [3].

In this paper, we present an approach towards ubiquitous access to contextualized medication-related guidance and decision support services targeting patients/citizens and healthcare professionals, respectively. Our work concerns the design and development of a "mobile component" aiming to exploit a novel decision support framework that was constructed in the scope of the PSIP EU-funded project [4].

Overall, the major challenge that we faced involved tailoring the designed services to the mobile terminal and the interactions that are favorable/enabled for/by modern smartphones. For example, we considered the technical features that these support, such as barcode scanning [5],

The research leading to these results has received funding from the European Community's Seventh Framework Program (FP7/2007-2013) under
Augmented Reality (AR) [6], and Near-Field Communication (NFC) tagging [7], in order to accurately identify the drugs of interest. Our aim was to avoid difficulties and errors in keyingin drug names by the user [8].

We illustrate the applicability and virtue of the proposed approach via a mobile application that has been particularly designed to meet the goal of ADE prevention given the patient's profile and a new drug of interest.

\section{TARGETED USERS AND REQUIREMENTS}

The target user groups of the mobile component are:

- Patients/citizens that are interested in accessing knowledge on potential ADEs according to their medical profile or health condition. This concerns information like diagnosis and laboratory examination results (if any), as well as current medication treatment for which ADE prevention rules may be applicable [4]. The user's interest may stem from checking simple drug-drug interactions to searching whether a drug could be appropriate for their medical condition. Evidently, the mobile component could be mostly attractive for user groups that are keen to mobile technologies, such as young people. Relatives of the patients could also use the application on their behalf.

- Healthcare professionals, e.g., nurses and medical doctors, who would like to have access to specialized decision support services for preventing ADEs for their patients at the point-of-care and, even more important, outside of the hospital environment where access to appropriate tools and knowledge is quite limited.

From a technical viewpoint, the fundamental requirement for using the mobile component is an (non-persistent) Internetconnected mobile terminal. In order to realize the option of guided identification of drugs through scanning the drug barcodes or through an AR-based approach, instead of prompting the user to type them, a camera is necessary as well as the installation of the appropriate software (either barcode reader, or AR libraries) in the mobile device. For NFC-based identification, the device has to support this technology and 
NFC tags have to be attached to the drug packages, introducing beyond cost, practical issues towards a generalizable solution.

From a user's viewpoint, basic familiarity with smartphone usage is beneficial. Nevertheless, through appropriate educational sessions on using the mobile component and its specific features, e.g., barcode scanning, the lack of such a familiarity can be overcome.

\section{ARCHITECTURAL DESIGN \& FUNCTIONAL ASPECTS}

In [4] we presented a knowledge engineering framework aiming to represent and manage various ADE patterns, with major focus on novel, rule-based signals obtained through knowledge discovery techniques applied on Electronic Health Records across Europe, and validated via a knowledge elicitation phase. The framework provides the basis for developing contextualized decision support services for ADE prevention through alerts and recommendations based on the assessment of the respective medical data [9].

In order to deploy the decision support services, a serviceoriented architecture (SOA) was defined. SOA offers a loosely coupled computing paradigm that has become a key ingredient of modern business applications and ICT infrastructures. In particular, a RESTful Web service was implemented in order to handle the requests from the mobile application. The service accepts the patient data along with contextual information (like filtering parameters and thresholds for rule firing [4]) in an XML-based format and queries the respective Clinical Decision Support System (CDSS). The CDSS response is also encoded in XML and returned to the corresponding application, which is hosted in our case in a mobile terminal.

A major design consideration was achieving a crossplatform implementation for the mobile component, aiming to accomplish its widest possible applicability and use. In addition, our development should take advantage of the smart tagging/identification technologies supported by modern smartphones to facilitate accurate and easy data entry.

The CDSS employed is able to process drug-related information based on the ATC (Anatomical Therapeutic Chemical) classification [10]. Thus, a barcode to ATC mapping was necessary to formulate the CDSS requests. This mapping is stored in a database containing also the commercial name of each drug. In addition, links to drug information portals have been proposed by domain experts, in order to provide further information as regards the drugs of interest.

Finally, as the employed CDSS aimed to offer its services in a variety of languages, multilinguality aspects were also an important requirement.

\section{USER INTERACTION DESIGN}

A major challenge for offering the functionality described above via a mobile terminal is confronting the limited key-in capabilities available for data input and the relatively small display for depicting and browsing the relevant information. Thus, a central design principle was to organize user interactions in logically concrete steps and in a wizard-like form, efficiently guiding this way the end-user. More specifically, the user is requested to enter data as regards the drugs that they took within the previous 5 days and the current day, laboratory examination results (if known/available) and potential diagnosis (if known/available); the above information is assessed against a drug of interest that could be potentially taken by the patient (e.g. upon a new drug prescription). These data are required for posing appropriate requests to the relevant CDSS. In addition, only the information that is relevant to ADE prevention rules was made available for user selection, e.g., only the laboratory examination parameters that take part as conditions in the elaborated ruleset and not an extensive list of such parameters.

The inherent user interface (UI) limitations that a typical mobile phone has (e.g., quite small display and difficulty in using the keyboard), may be eliminated by taking advantage of its integrated sensors that are almost always offered (e.g., the camera, by analyzing its input). In particular, in order to address the variety and complexity in providing input for the drugs of interest, the barcode available on each drug product package was used. Barcode technologies find a wide variety of applications contributing in the realization of the Internet of Things (IoT) [11], by providing links to information sources concerning the objects that they are attached to [12]. As the available drugs are thousands and different for each country, it is difficult for one to browse the respective large list in a mobile device and even write the exact commercial name of a specific drug due to the inherent difficulty in typing text. The barcode uniquely identifies the respective drug product, it corresponds to a series of digits (e.g. in France corresponds to only 7 digits, while in Denmark to 6 digits), and it is simple and quite error-free in comparison to the previous options described. Drug barcodes are typically linear or 1D.

For the identification of the $\operatorname{drug}(\mathrm{s})$ of interest, we also experimented with NFC tags (that we attached to the respective drug products), and an AR-based component enabling the user to define the $\operatorname{drug}(\mathrm{s})$ of interest by selecting the $\operatorname{drug}(\mathrm{s})$ that is(are) identified in the input scene of the smartphone camera. While these two approaches are more sophisticated compared to barcode scanning, we adopted the later option due to its simplicity, easier deployment (especially compared to the ARbased approach which requires the development of a database of drug product images) and lower cost (extra resources, like NFC tags, are not required).

\section{DEVELOPMENT ASPECTS}

For the development of the mobile component we adopted a rapid prototyping and testing approach. In continuous consultation with healthcare professionals, we started our experimentation with low-fidelity prototypes (using tools such as Justinmind Prototyper ${ }^{\circledR}$ ) and concluded with a fully functional mobile application.

Following a Web-based design, we achieved a platformindependent implementation, enabling this way the widest possible applicability and use (since a Web browser is typically incorporated in all modern mobile phones). Notably, the application was wrapped as a native Android/Java ME application. As the application content is dynamic, a serverside development was followed based on PHP scripting. 


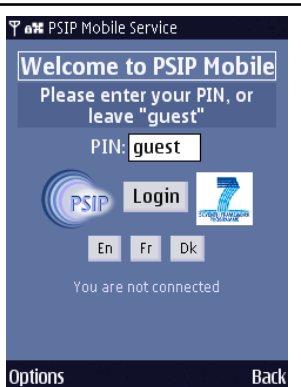

(b)

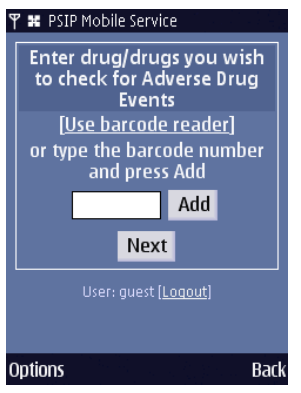

(d)

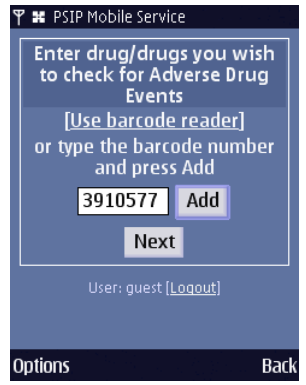

(f)

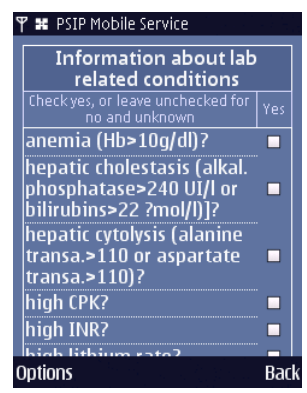

(h)

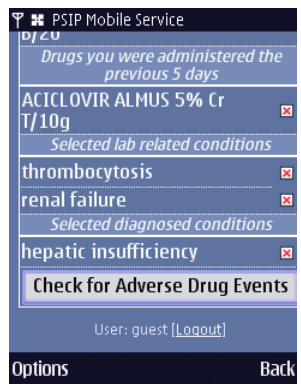

(j)

Figure 1. Sequential user interactions in the mobile app for ADE prevention.
A MySQL ${ }^{\mathrm{TM}}$ database was deployed to store/retrieve the dynamic content of the application that is determined according to the user interactions made.

The barcode reader employed is ZXing [13], a multi-format 1D/2D barcode image-processing library with clients for Android, iPhone, Java ME, etc. ZXing is an open-source solution, enabling its smooth integration in the developed Web application environment. ZXing provided good results in the initial testing phase and, thus, it was adopted as an optional part of the mobile patient component. Our application integrates ZXing, in the sense that when the user selects to use the barcode reader, if ZXing is already installed in the respective device it is launched or, in case not, the user is prompted to accept its download and installation. For the AR-based drug selection method, we experimented with the Qualcomm ${ }^{\circledR}$ Vuforia $^{\mathrm{TM}}$ SDK for Android [14].

The core CDSS module was implemented using the Drools Expert rule engine, which constitutes the core of the Drools rule management system. Drools is an open-source platform offering a forward and backward chaining inference based rule engine, which is based on an enhanced implementation of the Rete algorithm [15].

Finally, multilinguality aspects were addressed via the definition and use of an XML-based parameter file including the static content of the pages (labels) and database entries to host the dynamic content of the application (e.g. barcode-ATC mapping for drugs). Currently, the application is offered in Danish, English, French, and Greek for Android and Java ME.

\section{The Mobile APPLICATION FOR ADE PREVENTION}

Figure 1 depicts the basic user interactions enabled through the developed mobile application (due to space limitations, screens correspond to the Java ME application). First, the login screen prompts the user to enter his/her credentials and select the preferred language (Fig. 1(a)). Then, the user is prompted to enter his/her age, if logged in as "guest", or just confirm his/her age, if logged in via a registered account (Fig. 1(b)).

As a next step, the user has to enter the drugs of interest to be checked for ADEs (Fig. 1(c)), considering his/her existing patient drug intakes and medical history as defined in the following steps. Selecting "Use barcode reader", the barcode reader application is launched, if already installed in the mobile phone. If not, the user is prompted to download and install it. Alternatively, if the barcode reader application is unavailable or the phone does not support this functionality, the user is enabled to enter the barcode of the relevant drug in the respective input field. Pointing at the barcode of the drug of interest, the reader application identifies the respective code (Fig. 1(d)), and automatically uses this code to "fill-in" the input field depicted in Fig. 1(e), while the commercial name of the drug is presented to the end-user as a crosscheck reference (Fig. 1(f)). This step is repeated for all the drugs of interest.

Next, the user is prompted to select from a predefined list laboratory examination related conditions that he/she is aware of (Fig. 1(g)) and, similarly, diagnosis-related conditions (Fig. 1(h)). After an overall review of the provided input that is summarized in the next screen (Fig. 1(i)), the user queries the 
CDSS for ADEs related to his/her conditions by clicking on the "Check for Adverse Drug Events" button, moving this way to the final step (Fig. 1(j)). Depending on the user's profile (i.e., either patient or healthcare professional), the information provided for potential ADEs is appropriately tailored (simple/detailed information), while links to drug information portals are provided for the respective $\operatorname{drug}(\mathrm{s})$ of interest.

\section{DISCUSSION \& CONCLUSIONS}

This paper presented our work towards the design and development of a mobile component providing access to the decision support services developed in PSIP for ADE prevention [9]. Our design and development approach was primarily driven by the characteristics of the mobile terminals and the particular user requirements implied in the application context. Thus, the content of the application was eliminated to simple and rather short texts, while user interactions were organized through a step-by-step wizard.

In addition, we considered taking advantage of the sensing capabilities embodied in modern mobile devices, i.e., in particular, their camera accompanied with a barcode reader application to help the user (optionally) define the drugs of his/her interest instead of keying-in the respective codes. We also experimented with NFC tagging and AR-based drug identification, while we aim to investigate the potential of using speech-to-text mechanisms in future versions of the mobile component, so as to further eliminate typing data, which is laborious and error-prone in mobile terminals [8].

The presented work targets primarily patients wishing to access medication-guidance services via a mobile terminal, i.e., a smartphone or tablet. Especially mobile phones and the services they offer are widely used worldwide in everyday life, without requiring significant technical expertise from the endusers. Using a mobile phone is of course easier than using a computer, especially for the elder population, while young people are quite keen with this technology. In addition, offering guidance and decision support services via mobile terminals adds value in the sense of making these services available pervasively and ubiquitously, i.e., "anytime-anywhere". This is especially true for healthcare professionals, who would like to have access to specialized knowledge and decision support tools for ADE prevention not only at the point-of-care, but also outside the hospital environment where they may not easily access tools and knowledge sources.

Following a Web-based design, the mobile component is platform independent, as the only fundamental requirement of its use is having an Internet-connected mobile terminal with a Web browser that is typically pre-installed in such devices. For barcode tagging or the AR approach, an extra requirement is the availability of a built-in camera along with the respective software components.

The preliminary evaluation of the mobile component by healthcare professionals, illustrated that it may facilitate them to ubiquitously access specialized knowledge for $\mathrm{ADE}$ prevention and constitute a significant intervention for patient's safety. Nevertheless, a systematic evaluation with an adequate number of end-users is required to draw concrete conclusions as regards the impact of the proposed approach in medication safety, the usefulness of the application, and the level of user acceptance. This evaluation is currently under preparation.

In this regard, this work constitutes a proof-of-concept development towards pervasive and ubiquitous access to specialized decision support services and knowledge for ADE prevention. In this respect, our work differs from other efforts that typically concentrate on identifying drug-drug interactions (i.e., quite trivial knowledge that can be found in commercial drug databases) through mobile terminals and tagging technologies [16].

\section{REFERENCES}

[1] Institute of Medicine, "Preventing Medication Errors," The National Academic Press, US, 2007.

[2] M. Schachter, "The epidemiology of medication errors: how many, how serious?", Br. J. Clin. Pharmacol., vol. 67, no. 6, 2009, pp. 621-623.

[3] V. G. Koutkias, J. Niès, S. Jensen, N. Maglaveras, and R. Beuscart, "Patient Safety Informatics," Stud. Health. Technol. Inform., vol. 166, 2011, IOS Press.

[4] V. Koutkias et al., "Knowledge engineering for adverse drug event prevention: on the design and development of a uniform, contextualized and sustainable knowledge-based framework," J. Biomed. Inform., vol. 45, no. 3, 2012, pp. 495-506.

[5] E. Ohbuchi, H. Hanaizumi, and L. A. Hock, "Barcode readers using the camera device in mobile phones," In Proc. of the IEEE Int. Conf. on Cyberworlds, Nov. 18-20, 2004, pp. 260-265.

[6] G. Papagiannakis, G. Singh, and N. Magnenat-Thalmann, "A survey of mobile and wireless technologies for augmented reality systems," Comp. Anim. Virtual Worlds, vol. 9, no. 1, 2008, pp. 3-22.

[7] V. Coskun, K. Ok, and B. Ozdenizci, "Near Field Communication (NFC): From Theory to Practice," Wiley, 2012.

[8] J . H. Nichols et al., "Reducing medical errors through barcoding at the point of care," Clin. Leadersh. Manag. Rev., vol. 18, no. 6, 2004, pp. 328-334.

[9] V. Koutkias et al., "From adverse drug event detection to prevention: a novel clinical decision support framework for medication safety," Methods Inf. Med., (in press).

[10] The Anatomical Therapeutic Chemical classification system, Reference pages available at: http://www.whocc.no/atc/structure_and_principles/, last access: September 2014.

[11] J. Gubbi, R. Buyya, S. Marusica, and M. Palaniswami, "Internet of Things (IoT): a vision, architectural elements, and future directions," Future Generation Computer Systems, vol. 29, no. 7, 2013, pp. 16451660

[12] The Open Mobile Alliance (OMA), "White Paper on Mobile Codes", OMA-WP-MobileCodes-20080617-C, 17 Jun 2008, Available at: http://www.openmobilealliance.org/technical/release_program/docs/mob ilecodes/v1_0-20080617-c/oma-wp-mobilecodes-20080617-c.pdf, last access: September 2014.

[13] ZXing: Multi-format 1D/2D barcode image processing library with clients for Android, Java, Available at: https://github.com/zxing/zxing, last access: September 2014.

[14] Qualcomm $^{\circledast} \quad$ Vuforia $^{\mathrm{TM}} \quad$ SDK, Available at: https://developer.vuforia.com/resources/sdk/android, last access: September 2014.

[15] Drools, Available at: http://www.drools.org/, last access: September 2014.

[16] A. J. Jara et al., "A pharmaceutical intelligent information system to detect allergies and adverse drugs reactions based on Internet of Things," In Proc. of the 8th Int. Conf. on Pervasive Computing and Communications Workshops (PERCOM), Mar. 29-Apr. 2 2010, pp. 809-812. 